\title{
Magnetic Properties of the Phosphorous and Carbon with a Four and Five Bonds
}

\author{
Aibassov Yerkin Zhakenovich", Yemelyanova Valentina, Tussupbayev Nessipbay, Shakieva Tatyana, Bulenbayev \\ Maxat and Yerzhanova Zhadyra \\ Research Institute of New Chemical Technologies and Materials, Kazakh National University Al-Farabi, Almaty 005012, Kazakhstan
}

\begin{abstract}
The authors discovered a new magnetic properties of the phosphorous and carbon with a four and five bond, and stibine, bismuthine in organometallic chemistry. This allows to modify many of classical reactions by replacing with the nitrogen atoms to atoms of phosphorus, arsenic, antimony and bismuth. The authors have proposed a new mechanism for the possible reactions.
\end{abstract}

Key words: Organometallic chemistry, phosphorus, carbon, arsenic, antimony, bismuth.

\section{Introduction}

In recent years, many chemists have actively investigated nucleophilic organic halides phosphine with electrophilic hetero alkenes, allowing to obtain primary, secondary, tertiary and quaternary phosphines, selectively and in high yield. Most of these reactions are realized under mild conditions at atmospheric pressure phosphine.

Although the chemistry of organophosphorus compounds has devoted a considerable amount of books, but the reaction of organic compounds of arsenic, antimony and bismuth is very limited.

The purpose of this work is to search for new magnetic properties of the phosphorous and carbon with a four and five bond, and stibine, bismuthine in organometallic chemistry, carbon, arsenic, antimony and bismut.

\section{Theory}

Magnetic susceptibility $\chi$-a physical quantity that characterizes the relationship between the magnetic moment (magnetization) of the substance and the magnetic field in this matter.

The magnetic susceptibility is determined by the

\footnotetext{
*Corresponding author: Aibassov Yerkin Zhakenovich, professor, research fields: organic chemistry of $\mathrm{U}, \mathrm{Th}, \mathrm{As}, \mathrm{Sb}$, Bi.
}

ratio of the magnetization per unit volume of substance to the intensity of the magnetizing magnetic field.

$$
\chi=J / H
$$

where, $J$-the magnetization of matter under the influence of a magnetic field, $H$-magnetic field.

Real objects can have both positive and negative magnetic susceptibilities. Examples of substances with a negative susceptibility can serve diamagnetic - their magnetization in the direction opposite to the applied magnetic field. Positive susceptibility has, for example, paramagnetic and ferromagnetic materials.

The magnetic susceptibility of diamagnetic and paramagnetic small (it is negative for diamagnetic) and of the order of $10^{-4}-10^{-6}$, while it practically does not depend on the intensity of the applied magnetic field. Significant deviations are observed only in strong fields and low temperatures.

The magnetic permeability $\mu-\mathrm{a}$ physical quantity factor (depending on the properties of the medium), which characterizes the relationship between the magnetic induction $B$ and magnetic field strength $H$ in the matter. For different environments, this coefficient is different, so it is necessary to take into account $t$ the permeability specific environment (meaning its composition, condition, temperature).

The ratio between magnetic induction and magnetic 
field through the magnetic permeability is introduced as:

$$
\vec{B}=u \vec{H}
$$

Relative magnetic permeability $\mu$ is related with magnetic susceptibility $\chi$ ratio:

$$
\mu=1+\chi
$$

and Gaussian system permeability associated magnetic susceptibility $\chi$ ratio:

$$
\mu=1+4 \pi \chi
$$

Generally speaking, permeability depends on both the material properties, the magnitude, and direction of the magnetic field (and moreover the temperature, pressure and so on).

Also depends on the nature of variation of the field with time, in particular for the sinusoidal oscillations of the field - it depends on the frequency of oscillation (in this case the complex permeability is introduced to describe the effect of the medium on the phase shift of $B$ with respect to $H$ ). At sufficiently low frequencies (the speed of a small change in the field) it can usually be considered in this sense constant.

\section{Results and Discussion}

It is known that phosphorus has several modifications: white, red and black. Table 1 shows the properties modifications phosphorus.

As Table 1 shows, the transition from white to black phosphorus modification, magnetic susceptibility $\chi$ decreases with $-0.86 \times 10^{-9}$ to $-0.27 \times 10^{-9}$.

The authors have proposed to explain these facts from the point of view of the influence of the magnetic effect, which depends on the structure of phosphorus. In accordance with our proposal, the authors got unexpected results.

Quadruple bond is a diamond or coal or graphite! There just quadruple tuned!

Five-bond communication forms phosphorus. Then $\mathrm{P}_{2} \mathrm{H}_{2}$ molecule has the formula $\mathrm{HP} \equiv \mathrm{PH}$, where the phosphorus atom has five fold connection.

Table 2 shows the magnetic susceptibility molecules of ethane, ethylene and acetylene.

As can be seen from Table 2, with increasing amounts of hydrogen atoms from 2 to 6 , the length decreases from 15.4 to $12.0 \mathrm{~nm}$. Moreover, with decreasing amounts of hydrogen atoms from 2 to 6 , magnetic susceptibility decreases from $0.73 \times 10^{-8}$ to $0.43 \times 10^{-8} \mathrm{~m}^{3} / \mathrm{kg}$.

Thus, the stronger the bond, the less magnetic susceptibility in the molecule.

The authors suggested that if the phosphorus nucleus has a magnetic moment, the amount should be changed to white, red and black phosphorus. This should lead to the formation of new compounds of the phosphorous and carbon with a four and five bonds.

The authors propose to expand and modify the substitution reaction at the nitrogen atom of phosphorus, arsenic, antimony and bismuth.

Thus, the authors first proposed to expand the new magnetic properties of the phosphorous and carbon with a four and five bonds. The authors have proposed a new mechanism for the possible reactions of the phosphorous and carbon with a four and five bonds.

Table 1 Properties modifications phosphorus.

\begin{tabular}{lccc}
\hline Properties & White & Red & Black \\
\hline Density $\left(\mathrm{g} / \mathrm{cm}^{3}\right)$ & 1.828 & 2.310 & 2.700 \\
Temperature melting $\left({ }^{\circ} \mathrm{C}\right)$ & 44.14 & 593 & 1,000 \\
Magnetic susceptibility, $\chi \cdot 10^{-9}$ & -0.86 & -0.67 & -0.27 \\
\hline
\end{tabular}

Table 2 Magnetic susceptibility ethane, ethylene and acetylene.

\begin{tabular}{lll}
\hline Molecule & The length of the connection, $\mathrm{r}(\mathrm{nm})$ & Magnetic susceptibility, $\chi \cdot 10^{-8}\left(\mathrm{~m}^{3} / \mathrm{kg}\right)$ \\
\hline $\mathrm{C}_{2} \mathrm{H}_{6}$ & 15.4 & 0.73 \\
$\mathrm{C}_{2} \mathrm{H}_{4}$ & 13.4 & 0.48 \\
$\mathrm{C}_{2} \mathrm{H}_{2}$ & 12.0 & 0.43 \\
\hline
\end{tabular}




\section{Conclusions}

The authors have for the first time provided novel methods for the synthesis of organometallic compounds of possible phosphorus, carbon, arsenic, antimony, bismuth.

The mechanism of possible new compounds of phosphorus, carbon, arsenic, antimony, bismuth.

The proposed new methods of synthesis potential of organometallic compounds of phosphorus, arsenic, antimony, bismuth open new horizons to expand the potential application of biologically active substances, catalysts, pharmaceuticals preparations.

\section{Acknowledgments}

The authors would like to thank Lynn C.
Francesconi (Hunter College CUNY), Ruben M. Savizky (Columbia University, New York), Peter C. Burns (Notre Dame University, Indiana) and Chistopher L. Cahill (George Washington University) for discussion of the results.

\section{References}

[1] Furlani, E. P. 2001. Permanent Magnet and Electromechanical Devices: Materials, Analysis and Applications. New York: Academic Press.

[2] Griffiths, D. J. 1998. Introduction to Electrodynamics. 3rd ed. New Jersey: Prentice Hall.

[3] Kronmüller, H. 2007. Handbook of Magnetism and Advanced Magnetic Materials, 5 Volume Set. New York: John Wiley \& Sons.

[4] Tipler, P. 2004. Physics for Scientists and Engineers: Electricity, Magnetism, Light, and Elementary Modern Physics. 5th ed. New York: W H Freeman \& Co. 